\title{
Role of Radial Charges on the Angular Momentum of Electromagnetic Fields: Spin-3/2 Light
}

\author{
O. El Gawhary* \\ VSL Dutch Metrology Institute, Thijsseweg 112629 JA Delft, Netherlands \\ Optics Research Group, Imaging Physics Department, Delft University of Technology, \\ Van der Waalsweg 82628 CH Delft, Netherlands
}

T. Van Mechelen

Birck Nanotechnology Center and Purdue Quantum Center, Department of Electrical and Computer Engineering, Purdue University, West Lafayette 47907, Indiana, USA

H. P. Urbach

Optics Research Group, Imaging Physics Department, Delft University of Technology, Van der Waalsweg 8, 2628 CH Delft, Netherlands

(Received 10 April 2018; published 21 September 2018)

\begin{abstract}
Electromagnetic fields carry a linear and an angular momentum, the first being responsible for the existence of the radiation pressure and the second for the transfer of torque from electromagnetic radiation to matter. The angular momentum is considered to have two components, one due to the polarization state of the field, usually called spin angular momentum (SAM), and one due to the existence of topological azimuthal charges in the field phase profile, which leads to the orbital angular momentum (OAM). These two contributions to the total angular momentum of an electromagnetic field appear, however, to not be independent of each other, something which is described as spin-orbit coupling. Understanding the physics of this coupling has kept scientists busy for decades. Very recently it has been shown that electromagnetic fields necessarily carry also invariant radial charges that, as discussed in this Letter, play a key role in the angular momentum. Here we show that the total angular momentum consists in fact of three components: one component only dependent on the spin of the field, another dependent on the azimuthal charges carried by the field, and a third component dependent on the spin and the radial charges contained in the field. By properly controlling the number and coupling among these radial charges it is possible to design electromagnetic fields with a desired total angular momentum. Remarkably, we also discover fields with no orbital angular momentum and a spin angular momentum typical of spin-3/2 objects, irrespective of the fact that photons are spin-1 particles.
\end{abstract}

DOI: 10.1103/PhysRevLett.121.123202

Electromagnetic fields carry a linear and angular momentum, the first being responsible for the existence of the radiation pressure and the second for the transfer of torque from light to matter. Originally, most of the studies on this topic were concerned with linear momentum. After the work by Allen et al. [1], the interest on the angular momentum of light, its origin and effect on matter, has gradually grown through the years and currently represents an active area of investigation, especially in view of possible applications to high-capacity optical communications, novel metrology techniques, and classical and quantum imaging [2-6]. The

Published by the American Physical Society under the terms of the Creative Commons Attribution 4.0 International license. Further distribution of this work must maintain attribution to the author(s) and the published article's title, journal citation, and DOI. nature, and the origin, of the total angular momentum (TAM) for electromagnetic fields have been widely investigated and it is common opinion that there are essentially two sources for it: one due to the polarization state of the field (spin angular momentum or SAM, for short) and the other due to the presence of a helical wave front in the electromagnetic field (orbital angular momentum or OAM) [7]. The search for two such contributions has been mostly guided by analogies with mechanics and by the fact that the governing equation for monochromatic electromagnetic fields, under the paraxial regime, is formally equivalent to the Schrödinger equation for a quantum particle confined on a plane which is known to carry both types of angular momenta. Whether such formal division also applies to nonparaxial fields, i.e., to true solutions of Maxwell's equations, is still the subject of scientific investigation and debate. It could be that spin-orbit coupling is an inherent property of 
electromagnetic fields. After all, the governing equations for electromagnetism and quantum mechanics are in fact of fundamental different nature. This Letter intends to contribute to understanding the physics of this fundamental issue. More specifically, we make use of a recently introduced representation of a field in terms of Helmholtz natural modes, which allows us to identify two types of topological charges in a field: an azimuthal charge and a radial charge. As we are going to show in this Letter, radial topological charges are the physical objects that couple to the spin of the field. Once the main mechanism behind the generation of the total angular momentum (TAM) of a field is clear, we can show the existence of fields endowed with only a SAM contribution, which however corresponds to spin-3/2 particles, something surprising when dealing with electromagnetic fields.

In order to proceed, we need first to fix the notation used throughout the Letter. First of all, all fields depend on time by the factor $\exp (-i \omega t)$ where $\omega>0$. This factor is omitted from all formulae. As will be clear later on, it is convenient to express a generic monochromatic electromagnetic field, which propagates in the positive $z$ direction of a Cartesian reference frame, in terms of $s$ and $p$ polarized plane waves expansion as $\boldsymbol{E}(\boldsymbol{r})=\iint\left[A_{s}(\boldsymbol{\xi}) \hat{\boldsymbol{s}}(\boldsymbol{\xi})+A_{p}(\boldsymbol{\xi}) \hat{\boldsymbol{p}}(\boldsymbol{\xi})\right] \exp (i \boldsymbol{k} \cdot \boldsymbol{r}) d^{2} \boldsymbol{\xi}$, and $\boldsymbol{H}(\boldsymbol{r})=\sqrt{\varepsilon / \mu_{0}} \iint\left\{\left[-A_{s}(\boldsymbol{\xi}) \hat{\boldsymbol{p}}(\boldsymbol{\xi})+A_{p}(\boldsymbol{\xi}) \hat{\boldsymbol{s}}(\boldsymbol{\xi})\right] \exp (i \boldsymbol{k} \cdot \boldsymbol{r}) d^{2} \boldsymbol{\xi}\right\}$ with $\boldsymbol{k}=2 \pi \xi_{x} \hat{\boldsymbol{x}}+2 \pi \xi_{y} \hat{\boldsymbol{y}}+k_{z} \hat{\boldsymbol{z}}, \quad \mathbf{r}=x \hat{\boldsymbol{x}}+y \hat{\boldsymbol{y}}+z \hat{z}, \quad k_{z}=$ $\left[k^{2}-4 \pi^{2}\left(\xi_{x}^{2}+\xi_{y}^{2}\right)\right]^{1 / 2}, \quad$ and $\quad k^{2}=\left[4 \pi^{2}\left(\xi_{x}^{2}+\xi_{y}^{2}\right)+k_{z}^{2}\right]=$ $4 \pi^{2} / \lambda^{2}, \lambda$ being the wavelength. The functions $A_{s}(\xi)$ and $A_{p}(\xi)$ are the $s$ and $p$ components of the angular spectrum, respectively, and $\xi=\xi_{x} \hat{x}+\xi_{y} \hat{y}$ is the transverse spatial frequency vector. The unit vectors $\hat{\boldsymbol{s}}(\boldsymbol{\xi})$ and $\hat{\boldsymbol{p}}(\boldsymbol{\xi})$ are given by $\left[\xi_{y},-\xi_{x}, 0\right]\left(\xi_{x}^{2}+\xi_{y}^{2}\right)^{(-1 / 2)}$ and $\left[-\xi_{x} k_{z} / k,-\xi_{y} k_{z} / k\right.$, $\left.2 \pi\left(\xi_{x}^{2}+\xi_{y}^{2}\right) / k\right]\left(\xi_{x}^{2}+\xi_{y}^{2}\right)^{(-1 / 2)}$, respectively. Since we will only consider propagating fields, we neglect any contribution of evanescent waves to the field $\boldsymbol{E}(\boldsymbol{r})$; hence we have $4 \pi^{2}\left(\xi_{x}^{2}+\xi_{y}^{2}\right)<k^{2}$. From now on, we will be particularly interested in studying the properties of TAM in optical beams, paraxial or not, for which a mean direction of propagation, coinciding with the $z$ axis of a Cartesian reference frame, can still be identified. The time-averaged TAM can be written in the following way $[8,9]$ :

$$
\boldsymbol{J}=\frac{\varepsilon_{0} \mu_{0}}{2} \operatorname{Re}\left(\int\left[\mathbf{r} \times\left(\mathbf{E} \times \mathbf{H}^{\star}\right)\right] d \mathbf{r}\right)
$$

where the $\star$ symbol stands for complex conjugation. The majority of analyses and applications of SAM and OAM has always considered Laguerre-Gauss beams or other known solutions of the paraxial wave equation. The works which have addressed the case of nonparaxial fields suggested that for Maxwell fields the separation in SAM and OAM components in not possible [10-19]. This is the case, for instance, for tightly focused fields, or for light scattered by small objects. The reader interested in knowing more about the different scenarios where spin-orbit coupling plays a role is referred to the recent review work by Bliokh et al. [20]. In this Letter, we are not going to discuss the different physical interactions that can lead to a spin-orbit conversion, but we intend to investigate the physical origin behind such coupling. In order to do so, we will follow the common procedure of considering the angular momentum, and later on the energy, per unit length for a field propagating along the $z$ direction. This quantity reads

$$
\begin{aligned}
\mathfrak{J}_{z}= & \frac{\varepsilon_{0} \mu_{0}}{2} \operatorname{Re}\left\{\int\left[\mathbf{r} \times\left(\mathbf{E} \times \mathbf{H}^{\star}\right)\right]_{z} d x d y\right\} \\
= & \frac{\varepsilon_{0} \mu_{0}}{2} \operatorname{Re}\left\{\int \left[x\left(E_{z} H_{x}^{\star}-E_{x} H_{z}^{\star}\right)\right.\right. \\
& \left.\left.-y\left(E_{y} H_{z}^{\star}-E_{z} H_{y}^{\star}\right)\right] d x d y\right\} .
\end{aligned}
$$

Equation (2) can be put into a different, more insightful form by expressing it in Fourier space and in terms of polar coordinates, $\rho$ and $\varphi$ say, such that $\xi_{x}=\rho \cos \varphi$ and $\xi_{y}=\rho \sin \varphi$. This gives

$$
\begin{aligned}
\mathfrak{J}_{z}= & \operatorname{Re}\left\{\frac { \varepsilon _ { 0 } } { 2 i \omega } \int \left[\left(A_{s}^{\star} \frac{\partial A_{s}}{\partial \varphi}+A_{p}^{\star} \frac{\partial A_{p}}{\partial \varphi}\right)\right.\right. \\
& \left.\left.-\frac{(2 \pi)^{2}}{k} \frac{\rho^{2}}{k_{z}} A_{s}^{\star} A_{p}\right] \rho d \rho d \varphi\right\} .
\end{aligned}
$$

Equation (3) is an expression for the total angular momentum of surprising elegance and represents the first important result of this Letter. It shows that the TAM can in fact be expressed in terms of the sum of a $s$-polarized contribution (which we will call S-TAM), a $p$-polarized contribution (which we will denote as P-TAM) and a $s-p$ cross term (which we can denote as SP-TAM). Such a generic expression for the TAM of an electromagnetic field will now allow us to further investigate how spin and orbit couple to each other. In order to do this, we will use two main ingredients. First, we will decompose a generic field, solution of the scalar Helmholtz equation, in terms of Helmholtz natural modes (HNMs). Second, we will construct a solution of Maxwell's equations from a scalar solution of Helmholtz equation. Let us suppose we have a solution of the scalar Helmholtz equation $\nabla^{2} U(x, y, z)+$ $k^{2} U(x, y, z)=0$ where $k=2 \pi / \lambda$ and $U(x, y, z)=$ $\iint A\left(\xi_{x}, \xi_{y}\right) \exp \left[i 2 \pi\left(\xi_{x} x+\xi_{y} y\right)\right] \exp \left(i k_{z} z\right) d \xi_{x} d \xi_{y}$. Since we do not consider evanescent waves in the field, we can decompose the scalar angular spectrum $A\left(\xi_{x}, \xi_{y}\right)$ at $z=0$ in terms of orthogonal and propagation invariant modes, the Helmholtz natural modes [21], as

$$
A(\rho, \varphi)=\sum_{m, n} c_{m, n}\left[\frac{\exp \left(i 2 \pi m \lambda \sqrt{\frac{1}{\lambda^{2}}-\rho^{2}}\right)}{\left(\frac{1}{\lambda^{2}}-\rho^{2}\right)^{1 / 4}} \exp (\operatorname{in} \varphi)\right],
$$


with $m, n$ integers in the range $(-\infty, \infty)$. The resulting expression for the field $U(x, y, z)$ is

$$
\begin{aligned}
U(x, y, z)= & \sum_{m, n} c_{m, n} \exp (i n \theta) \\
& \times i^{n} \int_{0}^{1 / \lambda} \frac{\exp \left[i 2 \pi(m \lambda+z) \sqrt{\frac{1}{\lambda^{2}}-\rho^{2}}\right]}{\left(\frac{1}{\lambda^{2}}-\rho^{2}\right)^{1 / 4}} \\
& \times J_{n}\left(2 \pi \rho r_{\perp}\right) \rho d \rho,
\end{aligned}
$$

where $x=r_{\perp} \cos \theta, y=r_{\perp} \sin \theta$ and the coefficients $c_{m, n}$ are computed as

$$
\begin{aligned}
c_{m, n}= & \frac{\lambda}{2 \pi} \int_{0}^{2 \pi} \int_{0}^{1 / \lambda} \frac{A(\rho, \varphi)}{\left(\frac{1}{\lambda^{2}}-\rho^{2}\right)^{1 / 4}} \exp (-i n \varphi) \\
& \times \exp \left[-i 2 \pi m \lambda\left(\frac{1}{\lambda^{2}}-\rho^{2}\right)^{1 / 2}\right] \rho d \rho d \varphi .
\end{aligned}
$$

HNMs are orthogonal with respect to the scalar product and it is important to point out how each of the modes in a HNMs decomposition has finite energy and carries an azimuthal charge and a radial charge. Azimuthal charges appear in the form of a helical wave front and are due to the phase term $\exp (\operatorname{in} \varphi)$ in Eq. (4). Since the phase is not defined at the center of the coordinate system, the name of phase singularity is also a common way to refer to such phase profile. Azimuthal charges are responsible for the presence of an orbital angular momentum of light. Radial charges, in turn, appear in the form of Fresnel zones-like functional dependencies for the angular spectrum and are characterized by the phase profile $\exp \left(i 2 \pi m \lambda \sqrt{\left(1 / \lambda^{2}\right)-\rho^{2}}\right)$. The existence of a radial charge, preserved under free-space propagation, seems to have remained unnoticed until very recently $[21,22]$. The fundamental modes in real space consist of vortices in the azimuthal variable $\theta$ and integrals of Bessel functions in the radial variable $r_{\perp}$. The reader should notice that the only contribution to the field $U(x, y, z)$ on the optical axis $\left(r_{\perp}=0\right)$ comes from the coefficients $c_{m, 0}$, as expected. In fact, all the other terms would lead to an undefined phase at the points $(0,0, z)$, due to the presence of the vortex $\exp (\operatorname{in} \theta)$ in the phase profile, which would be not physical. In addition to that, we would also like to stress how, for any value of $n \neq 0$, the point $\rho=0$ in the integrand in Eq. (5) does not contribute to the value of the field $U(x, y, z)$ due to the presence of a zero of the $J_{n}\left(2 \pi \rho r_{\perp}\right)$ at $\rho=0$. As to a general procedure to build a vector field that is solution of Maxwell's equations from a scalar field $U$ solution of Helmholtz equation, a way is to combine two independent vectorial solutions of Maxwell's equations, namely $\boldsymbol{M}=$ $\nabla \times(\boldsymbol{v} U)$ and $\boldsymbol{N}=1 / k \nabla \times \boldsymbol{M}$, where $\boldsymbol{v}$ is a constant vector [23]. The field obtained in this way has, generally speaking, a position dependent polarization state, also sometimes denoted as local polarization state. In our case, we prefer to work with a field endowed with a global polarization state such that the state of polarization of the field components transverse to the mean direction of propagation is unequivocally defined. Such a field can be generated if one takes only the $\boldsymbol{M}$-type solution and further sets $\boldsymbol{v}=$ $1 / k\left[-i a_{2} \exp (i \alpha), i a_{1}, 0\right]$, where $a_{1}, a_{2}$, and $\alpha$ are realvalued constants that set the polarization state of the field. The resulting field reads

$$
\begin{aligned}
\boldsymbol{E}= & \iint A\left(\xi_{x}, \xi_{y}\right)\left\{a_{1} \frac{k_{z}}{k} \hat{x}+a_{2} \exp (i \alpha) \frac{k_{z}}{k} \hat{y}\right. \\
& \left.-\hat{z} \frac{1}{k}\left[a_{1} k_{x}+a_{2} k_{y} \exp (i \alpha)\right]\right\} \exp (i \boldsymbol{k} \cdot \mathbf{r}) d \xi_{x} d \xi_{y} .
\end{aligned}
$$

It can be checked that the field in Eq. (7) satisfies the transversality condition $\nabla \cdot \mathbf{E}=0$. Furthermore, it is easy to see that the transverse field $E_{x}(\boldsymbol{r}) \hat{x}+E_{y}(\boldsymbol{r}) \hat{y}$ is elliptically polarized, independently of position $\boldsymbol{r}$ [24]. In particular, if we set $a_{1}=a_{2}$ and $\alpha=0$, the transverse field component on the $x, y$ plane will be linearly polarized, while for $a_{1}=a_{2}$ and $\alpha= \pm \pi / 2$, it is circularly polarized. The field is also linearly polarized if $a_{1}=0$ or $a_{2}=0$. In the most general case $\left(a_{1} \neq a_{2}, \alpha \neq 0, \pi / 2\right)$, the field is elliptically polarized. From now on, we will consider the case of circular polarization, namely we will set $a_{1}=a_{2}=$ 1 and $\alpha= \pm \pi / 2$.

We have previously proven [see Eq. (3)] that the TAM can be expressed in terms of $s$ and $p$ components of the angular spectrum. For the field in Eq. (7), we can easily determine the expressions for $A_{s}(\boldsymbol{\xi})$ and $A_{p}(\boldsymbol{\xi})$, which read

$A_{s}\left(\xi_{x}, \xi_{y}\right)=A\left(\xi_{x}, \xi_{y}\right) \frac{k_{z}}{k}[\sin \varphi-\exp (i \alpha) \cos \varphi]$

$A_{p}\left(\xi_{x}, \xi_{y}\right)=-A\left(\xi_{x}, \xi_{y}\right)[\cos \varphi+\exp (i \alpha) \sin \varphi]$.

By inserting the expressions from Eq. (8) in Eq. (3), and further setting $\alpha= \pm \pi / 2$, we obtain

$$
\begin{aligned}
\mathfrak{J}_{z}^{( \pm \pi / 2)}= & \operatorname{Re}\left\{\frac { \varepsilon _ { 0 } } { 2 i \omega } \int _ { 0 } ^ { 2 \pi } \int _ { 0 } ^ { 1 / \lambda } \left[\left(i \sigma_{z}|A|^{2}+A^{\star} \frac{\partial A}{\partial \varphi}\right)\right.\right. \\
& \left.\left.\times\left(1+\frac{k_{z}^{2}}{k^{2}}\right)+\frac{(2 \pi)^{2}}{k^{2}} i \sigma_{z} \rho^{2}|A|^{2}\right] \rho d \rho d \varphi\right\}
\end{aligned}
$$

where, depending on the handedness of the polarization state, $\sigma_{z}= \pm 1$ denotes the component of the spin along the propagation direction. The energy per unit length $W$ of the field, $W=\varepsilon_{0} / 2 \iint\left[\left|A_{s}(\boldsymbol{\xi})\right|^{2}+\left|A_{p}(\boldsymbol{\xi})\right|^{2}\right] d^{2} \boldsymbol{\xi}$, can be expressed in terms of the scalar spectrum in the following way:

$$
W^{( \pm \pi / 2)}=\frac{\varepsilon_{0}}{2} \int_{0}^{2 \pi} \int_{0}^{1 / \lambda}|A|^{2}\left(1+\frac{k_{z}^{2}}{k^{2}}\right) \rho d \rho d \varphi .
$$

The ratio $\gamma=\mathfrak{\Im}_{z}^{( \pm \pi / 2)} / W^{( \pm \pi / 2)}$ between the TAM and the energy, per unit length, is 


$$
\begin{aligned}
\gamma= & \frac{\sigma_{z}}{\omega}+\frac{\operatorname{Re}\left\{\frac{1}{i \omega} \int_{0}^{2 \pi} \int_{0}^{1 / \lambda} A^{\star} \frac{\partial A}{\partial \varphi}\left(1+\frac{k_{z}^{2}}{k^{2}}\right) \rho d \rho d \varphi\right\}}{\int_{0}^{2 \pi} \int_{0}^{1 / \lambda}|A|^{2}\left(1+\frac{k_{z}^{2}}{k^{2}}\right) \rho d \rho d \varphi} \\
& +\frac{\operatorname{Re}\left\{\frac{\sigma_{z^{2}} \lambda^{2}}{\omega} \int_{0}^{2 \pi} \int_{0}^{1 / \lambda} \rho^{2}|A|^{2} \rho d \rho d \varphi\right\}}{\int_{0}^{2 \pi} \int_{0}^{1 / \lambda}|A|^{2}\left(1+\frac{k_{z}^{2}}{k^{2}}\right) \rho d \rho d \varphi} .
\end{aligned}
$$

The key step now to gain full insight on what controls each term in Eq. (11) is to expand $A(\rho, \varphi)$ in terms of Helmholtz natural modes, as in Eq. (4). This gives

$$
\begin{aligned}
\gamma= & \frac{\sigma_{z}}{\omega}+\frac{1}{\omega} \frac{\operatorname{Re}\left\{\sum_{m, n} \frac{4}{3} n\left|c_{m, n}\right|^{2}+\sum_{\substack{m, m^{\prime}, n \\
m \neq m^{\prime}}} n c_{m^{\prime}, n}^{\star} c_{m, n} f\left(m, m^{\prime}\right)\right\}}{\sum_{m, n} \frac{4}{3}\left|c_{m, n}\right|^{2}+\sum_{\substack{m, m^{\prime}, n \\
m \neq m^{\prime} \\
m, m^{\prime}, n}}^{\star} c_{m, n} f\left(m, m^{\prime}\right)} \\
& +\frac{\sigma_{z}}{\omega} \frac{\operatorname{Re}\left\{\sum_{m, n} \frac{2}{3}\left|c_{m, n}\right|^{2}-\sum_{\substack{m, m^{\prime}, n \\
m \neq m^{\prime}}} c_{m^{\prime}, n}^{\star} c_{m, n} f\left(m, m^{\prime}\right)\right\}}{\sum_{m, n} \frac{4}{3}\left|c_{m, n}\right|^{2}+\sum_{\substack{m, m^{\prime}, n \\
m \neq m^{\prime}}} c_{m^{\prime}, n}^{\star} c_{m, n} f\left(m, m^{\prime}\right)},
\end{aligned}
$$

where we defined the coupling function as

$$
f\left(m, m^{\prime}\right)=\left[\frac{1}{i 2 \pi\left(m-m^{\prime}\right)}+\frac{1}{2 \pi^{2}\left(m-m^{\prime}\right)^{2}}\right]
$$

Eq. (12) is the second fundamental result of this work. It shows that for the class of electromagnetic fields derived by

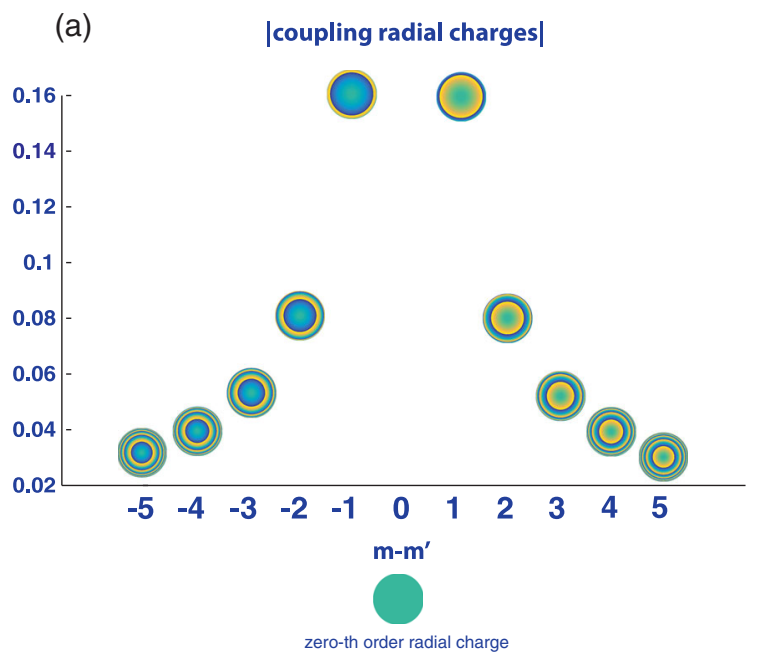

a scalar angular spectrum $A(\rho, \varphi)$, as in Eq. (7), with $a_{1}=a_{2}=1$ and $\alpha= \pm \pi / 2$, the TAM has three main contributions: a first term only dependent on the spin $\sigma_{z}$, a second one dependent on the azimuthal charges $n$ present in the field (the orbital part), and a last term dependent on the spin $\sigma_{z}$ and the radial charges $m$ contained in the field [25]. $\mathfrak{J}_{z} / W$ can be interpreted as if each photon in the field contributes to the angular momentum through a term $\sigma_{z} \hbar$ due to its spin, a term $n \hbar$ due to its orbital angular momentum, and a third term, again proportional to the spin $\sigma_{z}$ and function of the radial charges carried by the field. The last contribution, which is absent within the paraxial regime, is a special trait of the electromagnetic theory. If we look at the coupling function $f\left(m, m^{\prime}\right)$ in Eq. (13), we see it has a local form, since each individual radial charge effectively couples to only a limited number of neighboring charges, having radial indices $m$ not too different from each other and corresponding to the same azimuthal charge $n$. The local nature of the coupling can be better appreciated by looking at panel (a) of Fig. 1, where it is clear how each radial charge of radial index $m$ is effectively able to couple with few neighboring radial charges only. Since the mechanism behind the coupling is now clear, one can predict when it is relevant for applications and even properly design fields to obtain a specific value of the TAM. More importantly, if a field has only one radial charge $m$ for every single azimuthal charge $n$ (namely it is always $c_{m^{\prime}, n}^{\star} c_{m, n}=0$ when $m^{\prime} \neq m$ ),

(b)

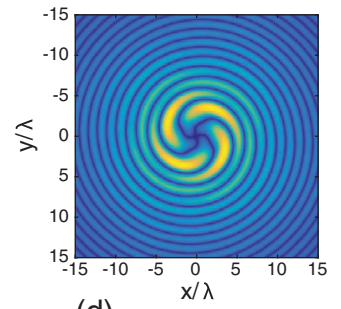

(d)

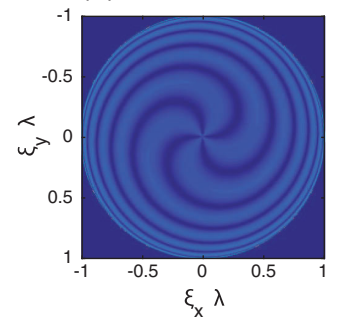

(c)
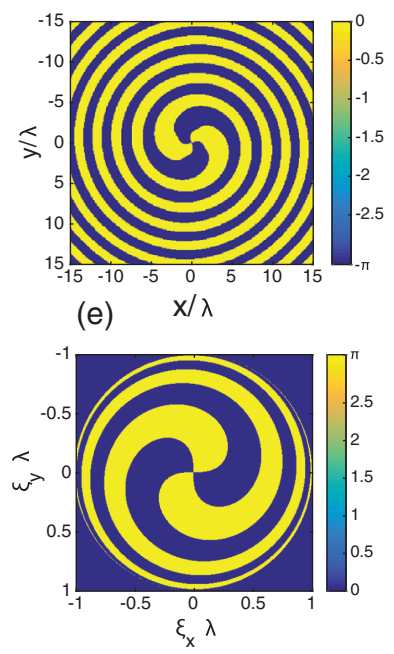

FIG. 1. Panel (a): Absolute value of the coupling function $f\left(m, m^{\prime}\right)=1 /\left[2 i \pi\left(m-m^{\prime}\right)\right]+1 /\left[2 \pi^{2}\left(m-m^{\prime}\right)^{2}\right]$ among neighboring radial modes. The curve refers to the coupling of the zeroth order radial charge (shown close to the origin of the $x$ axis in the graph) with the neighboring radial charges, of order $m \in[-5,5]$. On the $x$ axis the order of each charge is reported, while the phase profile of each charge is used as marker on the curve. Only radial charges corresponding to HNMs of same azimuthal charge $n$ can couple to each other. Panels (b), (c), (d), and (e): Example of spin-3/2 electromagnetic field. The field is derived by a scalar angular spectrum $A(\rho, \varphi)=\sin \left[2 \pi m \lambda \sqrt{\left(1 / \lambda^{2}\right)-\rho^{2}}+n \varphi\right] /\left[\left(1 / \lambda^{2}\right)-\rho^{2}\right]^{1 / 4}$. The absolute value, and phase, of the corresponding scalar field $U(x, y, z)$, at $z=0$, is given in panels (b) and (c), respectively. Panels (d) and (e) show the amplitude and phase of the corresponding scalar spectrum, $A$, within the circle of radius $1 / \lambda$ in Fourier space. The example corresponds to $m=3$ and $n=2$. The full electromagnetic field $\mathbf{E}$ associated to the angular spectrum $A$ is obtained through Eq. (7). More generally, any electromagnetic field carrying HNMs with no azimuthal charge (i.e., $n=0)$ will give a $\gamma=3 \sigma_{z} /(2 \omega)$. 
then there is no coupling among radial charges at all. In this specific case, Eq. (12) takes the remarkable form

$$
\gamma=\frac{3 \sigma_{z} / 2}{\omega}+\frac{1}{\omega} \frac{\sum_{m, n} n\left|c_{m, n}\right|^{2}}{\sum_{m, n}\left|c_{m, n}\right|^{2}}
$$

which shows a SAM typical of a spin-3/2 particle, something surprising when dealing with spin-1 particles, such as photons. Notice that the $3 / 2$ SAM is independent of the weighting coefficients $c_{m, n}$ and arises solely from the polarization state of the field. In addition, if the field does not have azimuthal charges (i.e., $n=0$ for all HNMs carried by the field), Eq. (14) gives $\gamma=3 \sigma_{z} /(2 \omega)$, which corresponds to the existence of electromagnetic fields only carrying a spin-3/2 angular momentum. Very recently, the possibility to generate light beams with half quantization of a total optical angular momentum has been discussed and experimentally tested within the paraxial regime [26]. The results obtained in the present work apply to electromagnetic fields, which are solutions of Maxwell's equations and stem from the coupling between radial charge and spin that takes place in the full nonparaxial regime. These results indicate that fractional-spin properties of light are of a much more fundamental nature and a direct consequence of the electromagnetic theory. As an example of an electromagnetic field that shows this unexpected spin-3/2 angular momentum, we consider the field with the following scalar angular spectrum

$$
A(\rho, \varphi)=\frac{\sin \left[2 \pi m \lambda \sqrt{\frac{1}{\lambda^{2}}-\rho^{2}}+n \varphi\right]}{\left(\frac{1}{\lambda^{2}}-\rho^{2}\right)^{1 / 4}} .
$$

The decomposition in HNMs of the angular spectrum in Eq. (15) only presents two terms different from zero, with coefficients $c_{m, n}=1 /(2 i)$ and $c_{-m,-n}=-1 /(2 i)$ [27]. Once this scalar spectrum is used to construct an electromagnetic field by using the recipe given in Eq. (8), one obtains exactly $\gamma=3 \sigma_{z} /(2 \omega)$. This is simple to see if one realizes that all products $c_{m^{\prime}, n}^{\star} c_{m, n}$ are zero, given that for different $m^{\prime}$ and $m$ there are no common azimuthal indices $n$. Additionally, it is important to point out that, although two azimuthal charges are present in the field, their contributions to the orbital angular momentum cancel with each other, leading to a field only carrying a spin angular momentum. In Figs. 1(b), 1(c), 1(d), and 1(e), we show amplitude and phase for the scalar angular spectrum $A$ for this field, along with amplitude and phase profiles, at $z=0$, of the corresponding scalar field $U$. To summarize, in this work we unfolded the different contributions to the total angular momentum of electromagnetic fields and showed how there are three main contributions: one due to spin, one due to the azimuthal charge carried by a field, and one which depends on the spin and the radial charges carried by the field. All this suggests that instead of talking of spin-orbit coupling, it would be more appropriate to consider coupling between spin, azimuthal charges, and radial charges in the field. A useful form for the total angular momentum has been obtained in terms of Helmholtz natural modes, which led to the discovery of fields for which only a spin-3/2 angular momentum exists. This is a surprising result, considering the quanta in an electromagnetic field, photons, are spin-1 particles. On an application perspective, the results presented here open novel routes to the control of light-matter interaction, optical metrology based on total angular momentum transfer and optical communications.

This work was partly funded through the Projects No. 14IND09 and No. 16ENG03 [European Metrology Programme for Innovation and Research (EMPIR)]. The EMPIR initiative is cofunded by the European Union Horizon 2020 research and innovation programme and the EMPIR participating States.

*o.elgawhary@tudelft.nl

[1] L. Allen, M. W. Beijersbergen, R. J. C. Spreeuw, and J. P. Woerdman, Phys. Rev. A 45, 8185 (1992).

[2] Quantum Imaging, edited by M. Kolobov (Springer, Singapore, 2007).

[3] Quantum Metrology, Imaging and Communication, edited by D. S. Simon, G. Jaeger, and A. V. Sergienko (Springer, Cham, 2017).

[4] Y. Yan, G. Xie, M. P. J. Lavery, H. Huang, N. Ahmed, C. Bao, Y. Ren, Y. Cao, L. Li, Z. Zhao, A. F. Molisch, M. Tur, M. J. Padgett, and A. E. Willner, Nat. Commun. 5, 4876 (2014).

[5] I. Bialynicki-Birula and Z. Bialynicka-Birula, J. Opt. 19, 125201 (2017).

[6] T. Van Mechelen and Z. Jacob, Optica 3, 118 (2016).

[7] We are referring here to the so-called intrinsic OAM. The extrinsic OAM, originating from a skew beam trajectory, is not of fundamental nature and will not be considered here.

[8] L. Allen, M. J. Padgett, and M. Babiker, in Progress in Optics, edited by E. Wolf (Elsevier, Amsterdam, 1999) XXXIX, pp. 291-372.

[9] C. Cohen-Tannoudji, J. Dupont-Roc, and G. Grynberg, Photons \& Atoms (Wiley-VHC, Weinheim, 2004).

[10] S. M. Barnett and L. Allen, Opt. Commun. 110, 670 (1994).

[11] K. Y. Bliokh, M. A. Alonso, E. A. Ostrovskaya, and A. Aiello, Phys. Rev. A 82, 063825 (2010).

[12] S. J. van Enk and G. Nienhuis, J. Mod. Opt. 41, 963 (1994).

[13] K. Y. Bliokh and F. Nori, Phys. Rep. 592, 1 (2015).

[14] I. Bialynicki-Birula and Z. Bialynicki-Birula, J. Opt. 13, 064014 (2011).

[15] L. Allen, M. Babiker, and W. L. Power, Opt. Commun. 112, 141 (1994).

[16] G. Gibson, J. Courtial, M. J. Padgett, M. Vasnetsov, V. Pasko, S. M. Barnett, and S. Franke-Arnold, Opt. Express 12, 5448 (2004).

[17] M. S. Soskin, V. N. Gorshkov, M. V. Vasnetsov, J. T. Malos, and N. R. Heckenberg, Phys. Rev. A 56, 4064 (1997). 
[18] S. M. Barnett, L. Allen, R. P. Cameron, C. R. Gilson, M. J. Padgett, F. C. Speirits, and A. M. Yao, J. Opt. 18, 064004 (2016).

[19] S. M. Barnett, M. Babiker, and M. J. Padgett, Phil. Trans. R. Soc. A 375, 20150444 (2017).

[20] K. Y. Bliokh, F. J. Rodriguez-Fortuno, F. Nori, and A. Zayats, Nat. Photonics 9, 796 (2015).

[21] O. El Gawhary, New J. Phys. 19, 013021 (2017).

[22] O. El Gawhary, Opt. Lett. 40, 2626 (2015).

[23] J. A. Stratton, Electromagnetic Theory (McGraw-Hill, New York and London, 1941).

[24] We emphasize that the $\mathbf{M}$ solutions are only chosen as a matter of convenience. The results obtained are general and valid regardless of this particular choice. If one chooses the $\mathbf{N}$ solutions, the roles of the $s$ and $p$ components for the electromagnetic field are simply interchanged. This has no effect on $\mathfrak{\Im}_{z}$ and the field energy $W$. A proof of this is provided in the Supplemental Material at http://link.aps.org/ supplemental/10.1103/PhysRevLett.121.123202.

[25] The reader should notice that the energy $W$ in the denominators of Eq. (12) is a real quantity, despite the apparent complex-valued expression. This can be easily proven by using the definitions of the coefficients $c_{m^{\prime}, n}^{\star}$ and $c_{m, n}$ and see what happens when $m$ and $m^{\prime}$ get replaced by $-m$ and $-m^{\prime}$

[26] K. Ballantine, J. F. Donegan, and P. R. Eastham, Sci. Adv. 2, e1501748 (2016).

[27] The reader might have noticed that the angular spectrum in Eq. (15) is multi-valued at $\rho=0$, for $n \neq 0$. The scalar field $U(x, y, z)$ corresponding to that spectrum is however singlevalued, since the point $\rho=0$ of the spectrum does not contribute to the field, due to the presence of a zero at $\rho=0$ carried by the function $J_{n}\left(2 \pi \rho r_{\perp}\right)$ as already discussed previously in the text. This means that in the practical realization of that angular spectrum one can replace the original spectrum by another one that vanishes at $\rho=0$, recovering in this way also a single-valued spectrum. The HNM decomposition of the two spectra remains the same, as the single point, where the two functions differ from each other, has zero measure and does not contribute to the integrals defining the coefficients $c_{m, n}$. 